\title{
Construction cost optimization of shallow foundation for sand soil in Indonesia
}

\author{
Abdul Azhim ${ }^{1, *}$ and Widjojo A. Prakoso ${ }^{1}$ \\ ${ }^{1}$ Department of Civil Engineering, Faculty of Engineering, Universitas Indonesia, Depok, Indonesia
}

\begin{abstract}
In designing foundation, there are three fundamental requirements namely: ultimate limit state (ULS), serviceability limit state (SLS), and economics. Generally, the ULS and SLS are the focus of the design process, while the cost factors are not explicitly considered. This paper deals with the three requirements with the minimum construction cost as the controlling objective. A wide range of sand density conditions are considered, while the typical ULS and SLS design checks available in the literature are employed. The optimization tool used is the Solver add-in function available in MS-Excel ${ }^{\mathrm{TM}}$. The effects of different construction cost structures in four Indonesia's provinces are examined in this paper. The purpose of this research is to be able in designing the shallow foundation with 3 aspects consideration at once, namely ULS, SLS and economic. On the other hand, this research is to analyse the sensitivity's factor which influence the design, namely work unit price, soil properties and design's requirements.
\end{abstract}

\section{Introduction}

In designing shallow foundation there are at least three fundamental requirements that need to be fulfilled, namely: ultimate limit state (ULS), serviceability limit state (SLS), and economics. In general, designing shallow foundation only focuses on ULS and SLS requirements, while the cost aspect is reviewed afterwards. Therefore, it is necessary to have a design approach that is explicitly able to consider economic aspects to construct foundations with low cost. This paper presents an optimization approach with Solver add-in function available in MS-Excel ${ }^{\mathrm{TM}}$ that explicitly accounts for the ULS, SLS, and economics requirements as the controlling objective and ensures an economically optimized design. The purpose of this research is to be able in designing the shallow foundation with 3 aspects consideration which is mentioned before at once. By doing optimization with solver on Microsoft Excel, it can be shown as well as the ULS design calculation's result which relates with factor of security, SLS design calculation's which relates with foundation settlement, and also cheapest foundation construction price. The output of this method is in the form of optimum design based on ULS's and SLS's requirements that is needed. The foundation construction minimum cost is then obtained from the work unit price's calculation to foundation optimum design.

\section{Conceptual framework}

Foundation is the lower part of building structure that functions to forward the upper structure loads to the supporting soil layer. Foundation with depths that are smaller or equal with its width are categorized as shallow foundations according to Terzaghi (1943).

$$
\mathrm{Df} \leq \mathrm{B} \text { or } \mathrm{Df} / \mathrm{B} \leq 1
$$

Bearing capacity is the capacity of soil to support the loads applied to the ground. The bearing capacity of soil is the maximum average contact pressure between the foundation and the soil which shouldn't produce shear failure in the soil.

Ultimate bearing capacity is the theoretical maximum pressure which can be supported without failure. Allowable bearing capacity is the ultimate bearing capacity divided by a factor of safety (FS).

Based on Terzaghi's bearing capacity theory, load $\mathrm{P}$ is resisted by shear stresses at edges of three zone under the footing and the overburden pressure $(q=\gamma D)$ above the footing. The first term in the equation is related to cohesion of the soil. The second term is related to the depth of the footing and overburden pressure. The third term is related to the width of the footing and the length of shear stress area.

$$
\mathrm{qu}=\mathrm{cNc}+\mathrm{qNq}+0,5 \gamma \mathrm{B} \mathrm{N \gamma}
$$

\footnotetext{
* Corresponding author: azhim23@gmail.com
} 
Equation (1) was derived for a strip footing and general shear failure where $\mathrm{Nc}, \mathrm{Nq}$, and $\mathrm{N} \gamma$ is Terzaghi's bearing capacity factors depend on soil friction angle $(\phi)$. However, Terzaghi's equations still has any shortcomings like they don't deal with rectangular foundations, the equations do not take into account the shearing resistance along the failure surface in soil above the bottom of the foundation, and the inclination of the load on the foundation is not considered (if exist). So, Meyerhoff suggested the following form of the general bearing capacity equation:

$q_{u}=c \lambda_{c s} \lambda_{c d} \lambda_{c i} N_{c}+q \lambda_{q s} \lambda_{q d} \lambda_{q i} N_{q}+\frac{1}{2} \lambda_{y s} \lambda_{y d} \lambda_{y i} i B N_{y}$

where:

$\mathrm{c}=$ cohesion of the underlying soil

$\mathrm{q}=$ effective stress at the level of the bottom of the foundation.

$\gamma=$ unit weight of the underlying soil

$\mathrm{B}=$ width of footing (diameter for a circular foundation)

$\mathrm{Nc}, \mathrm{Nq}$, and $\mathrm{N} \gamma=$ bearing capacity factors

$\lambda_{c s} \lambda_{c d} \lambda_{c i}=$ shape factor

$\lambda_{\mathrm{qqs}} \lambda_{\mathrm{qd}} \lambda_{\mathrm{qi}}=$ depth factor

$\lambda_{y s} \lambda_{y d} \lambda_{y i}=$ inclination factor

In this paper, research focused on sand soil which is cohesionless $(c=0)$ and a concentrically applied vertical load. So, the ultimate bearing capacity in Equation (2) can be simplified into:

$$
q_{u l t}=0,5 B \gamma^{\prime} N_{\gamma} \lambda_{\gamma s} \lambda_{\gamma d}+q^{\prime} N_{q} \lambda_{q s} \lambda_{q d}
$$

\section{Foundation construction cost estimate}

In the Economic Design Optimization of Foundations journal written by $\mathrm{Yu}$ Wang and Fred H. Kulhawy in 2008, Means (2006)a said that the shallow foundations cost construction on non-cohesive soil can be estimated by simplified unit price construction into five tasks, namely excavation, formwork, reinforcement, concreting, and compacted backfill.

All ULS and SLS Design value in MS-Excel ${ }^{\mathrm{TM}}$ Solver are obtained from calculation results to the formulas which is input on that cell. Formulas which is used based on the Terzaghi Soil Bearing Capacity's Theory. It is then researched by Vesic (1975). Below are the equation formulas that is inputted into the cell:

$$
\begin{gathered}
N_{q}=e^{\pi \tan \phi} \tan ^{2}\left(45+\frac{\phi}{2}\right) \\
q=\gamma \cdot D_{f} \\
\lambda_{q s}=1+(\tan \phi) \\
\lambda_{q d}=1+2 \tan \phi(1-\sin \phi)^{2}\left[\left(\frac{\pi}{180}\right) \tan ^{-1}\left(\frac{D f}{B}\right)\right] \\
N_{y}=2\left(N_{q}+1\right) \tan \phi \\
\lambda_{\gamma s}=0,6
\end{gathered}
$$

$$
\begin{gathered}
\lambda_{y d}=1 \\
\text { Bearing Capacity }=0,5 B \gamma^{\prime} N_{y} \lambda_{y s} \lambda_{y d}+q^{\prime} N_{q} \lambda_{q s} \lambda_{q d} \\
\text { Calculated } F S=\frac{q_{u l t}}{F / B L}
\end{gathered}
$$

Meanwhile the formulas that are become the value input on cell SLS Design category consists of equation formula $\beta_{z}$ value according to Whitman and Richart (1967) and equation formulas of decrease in foundation according to Poulos and Davis (1974), as of below:

$$
\begin{gathered}
\beta_{z}=-0,0017(L / B)^{2}+0,0597\left(\frac{L}{B}\right)+0,9843 \\
\text { Calculated Settlement }=\frac{F\left(1-v^{2}\right)}{\beta_{z} E \sqrt{B L}}
\end{gathered}
$$

\subsection{Cost optimization with Microsoft Excel Solver Add-Ins}

By inputting value of each soil property's parameter, unit price of five foundation activity works (excavation, formwork, reinforcement, concreting, and compacted backfill), and design requirements which is needed, then solver in Microsoft Excel will automatically find the solution of algorithm formula that is inputted so that results optimum dimension foundation includes the most minimum construction's cost.

But, in order to obtain the shallow foundation's dimension to be applied in the field, it is required to perform optimization limit input, as explained below: Calculated FSr $\geq$ Design FSr

Calculated $\delta \mathrm{r} \leq$ Design $\delta \mathrm{r}$

$$
\begin{aligned}
1,5 \mathrm{~m} & \leq \mathrm{D} \leq 2 \mathrm{~m} \\
1 \mathrm{~m} & \leq \mathrm{B} \leq 6 \mathrm{~m} \\
1 \mathrm{~m} & \leq \mathrm{L} \leq 6 \mathrm{~m}
\end{aligned}
$$

$\mathrm{B} / \mathrm{L}=1$ (rectangle-shape and round-shape)

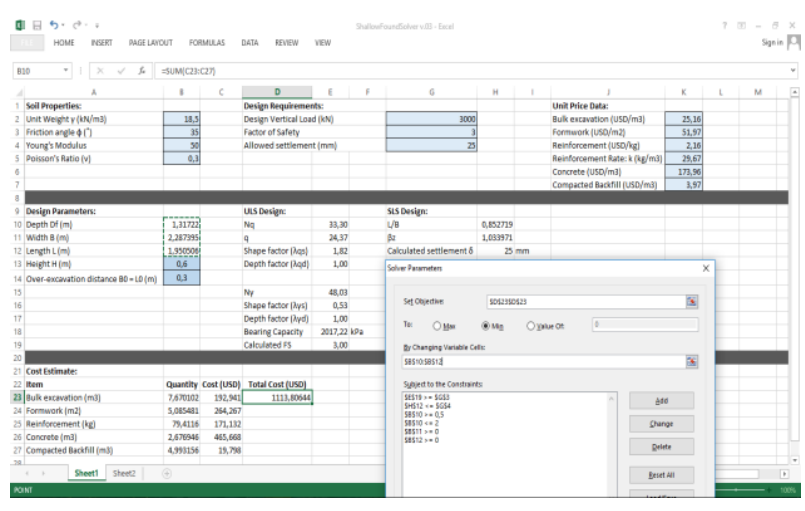

Fig. 1. Optimization's Result with Toolkit Solver in Microsoft Excel

By analyzing the sensitivity of various soil property factors, design requirements, and work unit prices, it can be seen what factors should be prioritized to do 
improvement so that the cost of the foundation construction becomes cheapest. Whether by repairing soil property, changing the design of the foundation, or moving the construction site to a location with a cheaper foundation work unit price.

\section{Sensitivity study}

Sensitivity study is done in order to know how big the influence of soil property's factor, foundation construction's cost, and design requirements of ULS and SLS to foundation construction minimum's cost is resulted. In order to know the sensitivity of 3 factors, then variation is done on one of the factors and perform uniformity on another factor.

\subsection{Effect of soil property}

Soil property variation is done by classify the soils generally based on relative density. At the very least, there are 5 classifications of sand soil based on relative density, which is shown below:

Table 1. Soil property variation

\begin{tabular}{|c|c|c|c|c|c|}
\hline \multirow{2}{*}{ Variation } & \multirow{2}{*}{$\begin{array}{c}\text { Type of } \\
\text { sand soil }\end{array}$} & $\begin{array}{c}\text { Unit } \\
\text { Weight } \\
(\mathrm{kN} / \mathrm{m} 3)\end{array}$ & $\begin{array}{c}\text { Friction } \\
\text { angle } \\
\left({ }^{\circ}\right)\end{array}$ & $\begin{array}{c}\text { Young's } \\
\text { Modulus } \\
(\mathrm{MPa})\end{array}$ & $\begin{array}{c}\text { Poisson's } \\
\text { Ratio }\end{array}$ \\
\hline 1 & $\begin{array}{c}\text { Very } \\
\text { Loose }\end{array}$ & 14 & 27,5 & 10 & 0,25 \\
\hline 2 & Loose & 15,5 & 30 & 15 & 0,25 \\
\hline 3 & Medium & 17,3 & 34 & 50 & 0,3 \\
\hline 4 & Solid/dense & 18,7 & 37 & 160 & 0,4 \\
\hline 5 & $\begin{array}{c}\text { Very } \\
\text { solid/dense }\end{array}$ & 19,3 & 40 & 250 & 0,4 \\
\hline
\end{tabular}

With assumption of unit price of foundation work activity is equal for each soil classification above and load is given with $3000 \mathrm{kN}, \mathrm{FS}=3$, and allowed settlement $=25 \mathrm{~mm}$. then, the results are:

Table 2. Optimization's result with variation of soil property

\begin{tabular}{|c|c|c|c|c|c|}
\hline $\begin{array}{l}\text { Type of } \\
\text { Sand Soil }\end{array}$ & \multicolumn{2}{|c|}{ Soil properties } & \multicolumn{2}{|c|}{ Dimension } & $\begin{array}{c}\text { Cost } \\
\text { (Rupiah) }\end{array}$ \\
\hline \multirow{4}{*}{ Very loose } & $\begin{array}{l}\text { Unit } \\
\text { Weight } Y \\
(\mathrm{kN} / \mathrm{m} 3)\end{array}$ & 14 & Depth (m) & 0,5 & \multirow{4}{*}{51.511 .600} \\
\hline & $\begin{array}{l}\text { Friction } \\
\text { angle } \phi\left(^{\circ}\right)\end{array}$ & 27,5 & Width (m) & 1,854 & \\
\hline & $\begin{array}{l}\text { Young's } \\
\text { Modulus } \\
(\mathrm{MPa})\end{array}$ & 10 & Length (m) & 30,112 & \\
\hline & $\begin{array}{l}\text { Poisson's } \\
\text { Ratio (v) }\end{array}$ & 0,25 & Area $\left(m^{2}\right)$ & 55,842 & \\
\hline & $\begin{array}{l}\text { Unit } \\
\text { Weight } Y \\
(\mathrm{kN} / \mathrm{m} 3)\end{array}$ & 15,5 & $\operatorname{Depth}(\mathrm{m})$ & 0,5 & \\
\hline Loose & $\begin{array}{l}\text { Friction } \\
\text { angle } \phi\left({ }^{\circ}\right)\end{array}$ & 30 & Width (m) & 1,262 & 25.147 .300 \\
\hline & $\begin{array}{l}\text { Young's } \\
\text { Modulus } \\
\text { (MPa) }\end{array}$ & 15 & Length (m) & 19,750 & \\
\hline & $\begin{array}{l}\text { Poisson's } \\
\text { Ratio (v) }\end{array}$ & 0,25 & Area $\left(\mathrm{m}^{2}\right)$ & 24,926 & \\
\hline Medium & $\begin{array}{l}\text { Unit } \\
\text { Weight } \gamma \\
(\mathrm{kN} / \mathrm{m} 3)\end{array}$ & 17,3 & Depth (m) & 1,484 & 6.199 .500 \\
\hline & $\begin{array}{l}\text { Friction } \\
\text { angle } \phi\left(^{\circ}\right)\end{array}$ & 34 & Width (m) & 2,852 & \\
\hline
\end{tabular}

\begin{tabular}{|c|c|c|c|c|c|}
\hline \multirow{3}{*}{$\begin{array}{c}\text { Type of } \\
\text { Sand Soil } \\
\end{array}$} & \multicolumn{2}{|c|}{ Soil properties } & \multicolumn{2}{|c|}{ Dimension } & \multirow{3}{*}{$\begin{array}{c}\text { Cost } \\
\text { (Rupiah) }\end{array}$} \\
\hline & $\begin{array}{l}\begin{array}{l}\text { Young's } \\
\text { Modulus } \\
(\mathrm{MPa})\end{array} \\
\end{array}$ & 50 & Length (m) & 1,615 & \\
\hline & $\begin{array}{l}\text { Poisson's } \\
\text { Ratio (v) }\end{array}$ & 0,3 & Area $\left(\mathrm{m}^{2}\right)$ & 4,607 & \\
\hline \multirow{4}{*}{ Solid/dense } & $\begin{array}{l}\text { Unit } \\
\text { Weight } Y \\
(\mathrm{kN} / \mathrm{m} 3)\end{array}$ & 18,7 & Depth (m) & 2 & \multirow{4}{*}{2.989 .000} \\
\hline & $\begin{array}{l}\text { Friction } \\
\text { angle } \phi\left(^{\circ}\right)\end{array}$ & 37 & Width (m) & 3,154 & \\
\hline & $\begin{array}{l}\text { Young's } \\
\text { Modulus } \\
\text { (MPa) }\end{array}$ & 160 & Length (m) & 0,422 & \\
\hline & $\begin{array}{l}\text { Poisson's } \\
\text { Ratio (v) }\end{array}$ & 0,4 & Area $\left(\mathrm{m}^{2}\right)$ & 1,331 & \\
\hline \multirow{4}{*}{$\begin{array}{c}\text { Very } \\
\text { solid/dense }\end{array}$} & $\begin{array}{l}\text { Unit } \\
\text { Weight } Y \\
(\mathrm{kN} / \mathrm{m} 3) \\
\end{array}$ & 19,3 & Depth (m) & 2 & \multirow{4}{*}{1.665 .100} \\
\hline & $\begin{array}{l}\text { Friction } \\
\text { angle } \phi\left(^{\circ}\right)\end{array}$ & 40 & Width (m) & 2,881 & \\
\hline & $\begin{array}{l}\text { Young's } \\
\text { Modulus } \\
\text { (MPa) }\end{array}$ & 250 & Length (m) & 0,116 & \\
\hline & $\begin{array}{l}\text { Poisson's } \\
\text { Ratio (v) }\end{array}$ & 0,4 & Area $\left(\mathrm{m}^{2}\right)$ & 0,333 & \\
\hline
\end{tabular}

Based on result above, it is known that there are significant differences between sand soil which is very loose and sand soil which is very dense. The most minimum cost is 51.5 millions IDR and keeps decreasing as the sand soil becomes more solid/dense. On the other hand, the least minimum cost is 1.665 millions IDR on sand soil that relatively very dense.

However, in order to know the largest factor that influence the amount of minimum cost, a study sensitivity is needed on every parameters. Sensitivity on each parameters can be known by performing addition and reduction of value on each parameters with $10 \%$ of parameters as the baseline.

Table 3. Input data of soil property sensitivity study

\begin{tabular}{|c|l|c|}
\hline Condition & \multicolumn{2}{|c|}{ Soil properties } \\
\hline \multirow{5}{*}{$+10 \%$} & Unit Weight $\gamma(\mathrm{kN} / \mathrm{m} 3)$ & 19,03 \\
\cline { 2 - 3 } & Friction angle $\phi\left(^{\circ}\right)$ & 37,4 \\
\cline { 2 - 3 } & Young's Modulus E $(\mathrm{MPa})$ & 55 \\
\cline { 2 - 3 } & Poisson's Ratio v & 0,33 \\
\hline \multirow{5}{*}{ Medium dense sand } & Unit Weight $\gamma(\mathrm{kN} / \mathrm{m} 3)$ & 17,3 \\
\cline { 2 - 3 } & Friction angle $\phi\left(^{\circ}\right)$ & 34 \\
\cline { 2 - 3 } & Young's Modulus E $(\mathrm{MPa})$ & 50 \\
\cline { 2 - 3 } & Poisson's Ratio v & 0,3 \\
\hline \multirow{5}{*}{$-10 \%$} & Unit Weight $\gamma(\mathrm{kN} / \mathrm{m} 3)$ & 15,57 \\
\cline { 2 - 3 } & Friction angle $\phi\left({ }^{\circ}\right)$ & 30,6 \\
\cline { 2 - 3 } & Young's Modulus E $(\mathrm{MPa})$ & 45 \\
\cline { 2 - 3 } & Poisson's Ratio v & 0,27 \\
\hline
\end{tabular}

By inputting parameters on solver, then the relation of each parameters with result of foundation construction minimum cost is obtained, as shown below: 


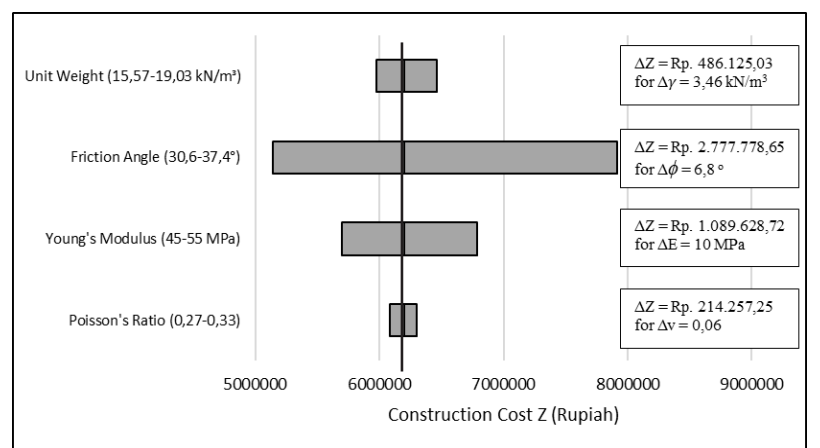

Fig. 2. Soil Property Sensitivity Influence on Foundation Minimum Cost

Sensitivity study can also be done by applying the typical value from coefficient of variation (COV) according to Phoon and Kulhawy (1999). Parameter determination based on $\mathrm{COV}$ is to make the $\gamma$ value and $\phi$ $\pm 10 \%$ from baseline, and $\mathrm{v}$ value and $\mathrm{E} \pm 50 \%$ from baseline so that the parameter value that is used as input data, which is shown below:

Table 4. Soil property input data based on COV

\begin{tabular}{|c|l|c|}
\hline Condition & \multicolumn{2}{|c|}{ Soil properties } \\
\hline \multirow{4}{*}{$\begin{array}{c}\gamma, \phi+10 \% \\
v, E+50 \%\end{array}$} & Unit Weight $\gamma(\mathrm{kN} / \mathrm{m} 3)$ & 19,03 \\
\cline { 2 - 3 } & Friction angle $\phi\left(^{\circ}\right)$ & 37,4 \\
\cline { 2 - 3 } & Young's Modulus E (MPa) & 75 \\
\cline { 2 - 3 } & Poisson's Ratio v & 0,45 \\
\hline \multirow{4}{*}{$\begin{array}{c}\text { Medium dense sand } \\
\end{array}$} & Unit Weight $\gamma(\mathrm{kN} / \mathrm{m} 3)$ & 17,3 \\
\cline { 2 - 3 } & Friction angle $\phi\left(^{\circ}\right)$ & 34 \\
\cline { 2 - 3 } & Young's Modulus E $(\mathrm{MPa})$ & 50 \\
\cline { 2 - 3 } $\begin{array}{c}\gamma, \phi-10 \% \\
v, E-50 \%\end{array}$ & Poisson's Ratio v & 0,3 \\
\hline & Unit Weight $\gamma(\mathrm{kN} / \mathrm{m} 3)$ & 15,57 \\
\cline { 2 - 3 } & Friction angle $\phi\left(^{\circ}\right)$ & 30,6 \\
\cline { 2 - 3 } & Young's Modulus E $(\mathrm{MPa})$ & 25 \\
\cline { 2 - 3 } & Poisson's Ratio v & 0,15 \\
\hline
\end{tabular}

By inputting parameters as shown above, then the relation of parameters with foundation construction minimum cost is obtained, as shown below:



Fig. 3. Soil Property Sensitivity Influence (COV) on Foundation Minimum Cost

From the 2 types of soil property sensitivity study which is shown above, can be known that parameter which becomes the important key to shallow foundation construction minimum cost on sand soil is the friction angle's parameter and Young's modulus. Therefore, in order to improve the soil parameter, friction angle and Young's modulus should be more important to improved.

\subsection{Effect of unit price}

Foundation work unit price's sensitivity study is done by making the variation of unit price's value from the five foundation work activity by taking examples of work unit price from four different cities, as explained below:

Table 5. Foundation work unit price variation in four different province

\begin{tabular}{|c|c|c|c|c|c|}
\hline \multirow[b]{2}{*}{ Work task } & \multirow[b]{2}{*}{ Details } & \multicolumn{4}{|c|}{ Cost per unit (Rupiahs) } \\
\hline & & $\begin{array}{c}\text { Suma- } \\
\text { tera } \\
\text { Barat }\end{array}$ & $\begin{array}{l}\text { Jawa } \\
\text { Timur }\end{array}$ & $\begin{array}{c}\text { Kali- } \\
\text { mantan } \\
\text { Tengah }\end{array}$ & $\begin{array}{c}\text { DKI } \\
\text { Jakarta }\end{array}$ \\
\hline Excavation & $\begin{array}{l}\text { Soil excavation } \\
\text { until } 2 \mathrm{~m} \text { depth }\end{array}$ & 73.000 & 86.500 & 117.200 & 143.700 \\
\hline Formwork & $\begin{array}{l}\text { Foundation } \\
\text { Formwork }\end{array}$ & $\begin{array}{c}348.90 \\
0\end{array}$ & 256.300 & 202.800 & 25.600 \\
\hline $\begin{array}{c}\text { Reinforceme } \\
\text { nt }\end{array}$ & $\begin{array}{l}\text { Threaded steel } \\
\text { bar }(1 \mathrm{bar} 12 \mathrm{~m} \text {, } \\
7850 \mathrm{~kg} / \mathrm{m} 3)\end{array}$ & 13.500 & 12.500 & 16.700 & 8.700 \\
\hline $\begin{array}{c}\text { Reinforceme } \\
\text { nt rate }\end{array}$ & - & 29,67 & 29,67 & 29,67 & 29,67 \\
\hline Concrete & $\begin{array}{l}\text { Standart Ready } \\
\text { Mix K250 }\end{array}$ & $\begin{array}{c}963.80 \\
0\end{array}$ & $\begin{array}{c}1.192 .70 \\
0\end{array}$ & $\begin{array}{c}1.241 .40 \\
0\end{array}$ & 872.000 \\
\hline $\begin{array}{c}\text { Compacted } \\
\text { Backfill }\end{array}$ & $\begin{array}{l}\text { Compacted } \\
\text { sand backfill }\end{array}$ & 30.300 & 189.200 & 69.200 & 84.800 \\
\hline
\end{tabular}

Variation above results the shallow foundation construction's minimum cost, as shown below:

Table 6. Optimization's result with variation of unit price data

\begin{tabular}{|c|c|c|c|c|}
\hline Province & \multicolumn{2}{|c|}{ Dimension } & Settlement & Cost \\
\hline \multirow{4}{*}{$\begin{array}{l}\text { DKI } \\
\text { Jakarta }\end{array}$} & Depth (m) & 2 & \multirow{4}{*}{$7 \mathrm{~mm}$} & \multirow{4}{*}{2.378 .400} \\
\hline & Width (m) & 1,274 & & \\
\hline & Length (m) & 1 & & \\
\hline & Area $\left(\mathrm{m}^{2}\right)$ & 1,274 & & \\
\hline \multirow{4}{*}{$\begin{array}{c}\text { Sumatera } \\
\text { Barat }\end{array}$} & Depth (m) & 2 & \multirow{4}{*}{$7 \mathrm{~mm}$} & \multirow{4}{*}{2.392 .100} \\
\hline & Width (m) & 1,274 & & \\
\hline & Length (m) & 1 & & \\
\hline & Area $\left(\mathrm{m}^{2}\right)$ & 1,274 & & \\
\hline \multirow{4}{*}{$\begin{array}{l}\text { Jawa } \\
\text { Timur }\end{array}$} & Depth (m) & 2 & \multirow{4}{*}{$7 \mathrm{~mm}$} & \multirow{4}{*}{2.877 .400} \\
\hline & Width (m) & 1,274 & & \\
\hline & Length (m) & 1 & & \\
\hline & Area $\left(\mathrm{m}^{2}\right)$ & 1,274 & & \\
\hline
\end{tabular}




\begin{tabular}{|c|c|c|c|c|}
\hline Province & \multicolumn{2}{|c|}{ Dimension } & $\begin{array}{c}\text { Settlement } \\
(\mathbf{m m})\end{array}$ & $\begin{array}{c}\text { Cost } \\
\text { (Rupiahs) }\end{array}$ \\
\hline \multirow{4}{*}{$\begin{array}{c}\text { Kalimantan } \\
\text { Tengah }\end{array}$} & Depth $(\mathrm{m})$ & 2 & & \\
\cline { 2 - 3 } & Width(m) & 1,274 & \multirow{3}{*}{$7 \mathrm{~mm}$} & \multirow{2}{*}{2.589 .600} \\
\cline { 2 - 3 } & Length $(\mathrm{m})$ & 1 & & \\
\cline { 2 - 3 } & Area $\left(\mathrm{m}^{2}\right)$ & 1,274 & & \\
\hline
\end{tabular}

The result above shows that the area with the relatively low foundation work unit price will also tend to produce the low cost of foundation construction. DKI Jakarta with the lowest foundation work unit price produces the lowest construction minimum cost. Meanwhile, Jawa Timur with the highest foundation work unit price produces the highest cost amongst other provinces.

\subsection{Effect of design requirement}

Sensitivity study is also done by make foundation design's requirements vary and by perform uniformity on unit price of foundation work and sand soil property.

Table 6. Design requirement's parameter on variation data input

\begin{tabular}{|l|l|}
\hline \multicolumn{1}{|c|}{ Parameters } & \multicolumn{1}{c|}{ Value } \\
\hline $\begin{array}{l}\text { Design Vertical Load } \\
\mathrm{kN})\end{array}$ & $\begin{array}{l}150,500,1000,1500,2000 \\
\text { dan 3000 kN }\end{array}$ \\
\hline Factor of Safety & 2,3, dan 4 \\
\hline $\begin{array}{l}\text { Allowed Settlement } \\
(\mathrm{mm})\end{array}$ & $10 \mathrm{~mm}-25 \mathrm{~mm}(\Delta \delta=3 \mathrm{~mm})$ \\
\hline
\end{tabular}

From design parameter variation which is done, the results as shown below:

Table 7. Correlation between vertical load variation and foundation minimum cost

\begin{tabular}{|c|c|c|c|c|}
\hline $\begin{array}{c}\text { Design } \\
\text { Vertical }\end{array}$ & \multicolumn{2}{|c|}{ Dimension } & $\begin{array}{l}\text { Calculated } \\
\text { Settlement }\end{array}$ & $\begin{array}{c}\text { Cost } \\
\text { (Rupiah) }\end{array}$ \\
\hline \multirow{4}{*}{$150 \mathrm{kN}$} & $\begin{array}{l}\text { Depth } \\
(\mathrm{m})\end{array}$ & 1,5 & \multirow{4}{*}{$1 \mathrm{~mm}$} & \multirow{4}{*}{1.773 .700} \\
\hline & $\begin{array}{l}\text { Width } \\
\text { (m) }\end{array}$ & 1 & & \\
\hline & $\begin{array}{l}\text { Length } \\
\text { (m) }\end{array}$ & 1 & & \\
\hline & Area $\left(\mathrm{m}^{2}\right)$ & 1 & & \\
\hline \multirow{4}{*}{$500 \mathrm{kN}$} & $\begin{array}{l}\text { Depth } \\
\text { (m) }\end{array}$ & 1,5 & \multirow{4}{*}{$3 \mathrm{~mm}$} & \multirow{4}{*}{1.773 .700} \\
\hline & $\begin{array}{l}\text { Width } \\
\text { (m) }\end{array}$ & 1 & & \\
\hline & $\begin{array}{l}\text { Length } \\
\text { (m) }\end{array}$ & 1 & & \\
\hline & Area $\left(\mathrm{m}^{2}\right)$ & 1 & & \\
\hline \multirow{4}{*}{$1000 \mathrm{kN}$} & $\begin{array}{l}\text { Depth } \\
\text { (m) }\end{array}$ & 1,867 & \multirow{4}{*}{$5 \mathrm{~mm}$} & \multirow{4}{*}{1.915 .600} \\
\hline & $\begin{array}{l}\text { Width } \\
\text { (m) }\end{array}$ & 1 & & \\
\hline & $\begin{array}{l}\text { Length } \\
\text { (m) }\end{array}$ & 1 & & \\
\hline & Area $\left(\mathrm{m}^{2}\right)$ & 1 & & \\
\hline
\end{tabular}

\begin{tabular}{|c|c|c|c|c|}
\hline $\begin{array}{c}\text { Design } \\
\text { Vertical }\end{array}$ & \multicolumn{2}{|c|}{ Dimension } & $\begin{array}{l}\text { Calculated } \\
\text { Settlement }\end{array}$ & $\begin{array}{c}\text { Cost } \\
\text { (Rupiah) }\end{array}$ \\
\hline \multirow{4}{*}{$1500 \mathrm{kN}$} & $\begin{array}{l}\begin{array}{l}\text { Depth } \\
(\mathrm{m})\end{array} \\
\end{array}$ & 2 & \multirow{4}{*}{$7 \mathrm{~mm}$} & \multirow{4}{*}{2.378 .400} \\
\hline & Width(m) & 1,274 & & \\
\hline & $\begin{array}{l}\text { Length } \\
(\mathrm{m})\end{array}$ & 1 & & \\
\hline & Area $\left(\mathrm{m}^{2}\right)$ & 1,274 & & \\
\hline \multirow{4}{*}{$2000 \mathrm{kN}$} & $\begin{array}{l}\text { Depth } \\
(\mathrm{m})\end{array}$ & 2 & \multirow{4}{*}{$8 \mathrm{~mm}$} & \multirow{4}{*}{ 2.805.000, } \\
\hline & $\begin{array}{l}\text { Width } \\
(\mathrm{m})\end{array}$ & 1,557 & & \\
\hline & $\begin{array}{l}\text { Length } \\
(\mathrm{m})\end{array}$ & 1 & & \\
\hline & Area $\left(\mathrm{m}^{2}\right)$ & 1,557 & & \\
\hline \multirow{4}{*}{$3000 \mathrm{kN}$} & $\begin{array}{l}\text { Depth } \\
(\mathrm{m})\end{array}$ & 2 & \multirow{4}{*}{$11 \mathrm{~mm}$} & \multirow{4}{*}{3.585 .500} \\
\hline & $\begin{array}{l}\text { Width } \\
\text { (m) }\end{array}$ & 2,076 & & \\
\hline & $\begin{array}{l}\text { Length } \\
(\mathrm{m})\end{array}$ & 1 & & \\
\hline & Area $\left(\mathrm{m}^{2}\right)$ & 2,076 & & \\
\hline
\end{tabular}

Table 8. Correlation between safety factor variation and foundation minimum cost

\begin{tabular}{|c|c|c|c|c|}
\hline $\begin{array}{c}\text { Factor } \\
\text { of Safety }\end{array}$ & \multicolumn{2}{|c|}{ Dimension } & $\begin{array}{l}\text { Calculated } \\
\text { Settlement }\end{array}$ & $\begin{array}{c}\text { Cost } \\
\text { (Rupiah) }\end{array}$ \\
\hline \multirow{4}{*}{2} & Depth (m) & 1,867 & \multirow{4}{*}{$8 \mathrm{~mm}$} & \multirow{4}{*}{1.915 .600} \\
\hline & Width (m) & 1 & & \\
\hline & Length (m) & 1 & & \\
\hline & Area $\left(\mathrm{m}^{2}\right)$ & 1 & & \\
\hline \multirow{4}{*}{3} & Depth (m) & 2 & \multirow{4}{*}{$7 \mathrm{~mm}$} & \multirow{4}{*}{2.378 .400} \\
\hline & Width (m) & 1,274 & & \\
\hline & Length (m) & 1 & & \\
\hline & Area $\left(\mathrm{m}^{2}\right)$ & 1,274 & & \\
\hline \multirow{4}{*}{4} & Depth (m) & 2 & \multirow{4}{*}{$6 \mathrm{~mm}$} & \multirow{4}{*}{2.805 .000} \\
\hline & Width (m) & 1,557 & & \\
\hline & Length $(\mathrm{m})$ & 1 & & \\
\hline & Area $\left(\mathrm{m}^{2}\right)$ & 1,557 & & \\
\hline
\end{tabular}

From the optimization process as given above, can be known that the relation between vertical load design and the cost need is linear. If the value of vertical load design increased, so the cost. On the other hand, if the vertical load design increased, the calculation of settlement possibility also relatively increased.

The larger foundation safety factor, will also increase the foundation construction minimum cost. However, the settlement possibility will be small.

\section{Conclusion}

Solver add-ins in Microsoft Excel can be a tool in order to know and to analyze the shallow foundation construction minimum's cost and the parameter sensitivities that influence the cost. 
The more loose relative density on sand soil, the higher the foundation construction's cost. Meanwhile, the more dense relative density on sand soil, the lower the construction's cost. The biggest factor that influences foundation construction's cost is the friction angle and young's modulus.

An area with relatively low foundation work unit price is tend to produce the low foundation construction's cost. If the value of vertical load design increased, so the cost. On the other hand, if the vertical load design increased, the calculation of settlement possibility also relatively increased.

The larger foundation safety factor, will also increase the foundation construction minimum cost. However, the settlement possibility will be small.

The results of this study indicate that the optimization procedure could optimize the foundation construction costs while fulfilling the ULS and SLS design requirements. The optimization process could also consider the variability of soil properties.

The author gratefully acknowledge the part support provided through the research grant PUPT by Minister of Research, Technology, and Higher Education through Universitas Indonesia under the contract No 513/UN2.R3.1/HKP.05.00/2018.

\section{References}

1. Wang, Yu, \& H.Kulhawy, Fred. Economic Design Optimization of Foundations. ASCE: Journal of Geotechnical and Geoenvironmental Engineering. (2008)

2. Endah, Noor, \& B. Mochtar, Indrasurya. Mekanika Tanah (Prinsip-prinsip Rekayasa Geoteknis) Jilid 2. USA: Braja M. Das. (1993)

3. Fylstra, Daniel, Lasdon, Leon, Watson, John, \& Waren, Allan. Design and Use of The Microsoft Excel Solver. INTERFACES, Vol.28, No.5, pp. 2955. (1998)

4. Construction Ministry of China (CMC). (1995). dalam Wang, Yu, \& H. Kulhawy, Fred. Economic Design Optimization of Foundations. ASCE: Journal of Geotechnical and Geoenvironmental Engineering. (2008)

5. Indonesia, Peraturan Menteri Pekerjaan Umum tentang Pedoman Analisis Harga Satuan Pekerjaan Bidang Pekerjaan Umum, PRT. No. 11. (2013)

6. Basari, Rambang. Jurnal Harga Satuan Bahan Bangunan Konstruksi dan Interior Edisi XXXII. Jakarta: Yayasan Pandu Bangun Persada Nusantara Batavia. (2013)

7. Naval Facilities Engineering Command (NAVFAC). Soil Mechanics Design Manual 7.01. 200 Stovall Street Alexandria, Virginia: NAVFAC. (1986) 